\title{
miR-224-5p protects dental pulp stem cells from apoptosis by targeting Rac1
}

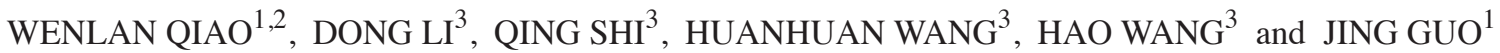 \\ ${ }^{1}$ Shandong Provincial Key Laboratory of Oral Tissue Regeneration, Department of Orthodontics, School of Stomatology, \\ Shandong University; ${ }^{2}$ Department of Stomatology, Qilu Hospital, and Institute of Stomatology, Shandong University; \\ ${ }^{3}$ Department of Cryomedicine Laboratory, Qilu Hospital of Shandong University, Jinan, Shandong 250012, P.R. China
}

Received November 28, 2018; Accepted July 12, 2019

DOI: $10.3892 /$ etm.2019.8213

\begin{abstract}
Dental pulp stem cells (DPSCs) are reported to be enriched in stem/progenitor cells, however to the best of our knowledge they have yet to be well documented and characterized. In the present study, in order to characterize DPSCs and the effect of microRNAs (miRs/miRNAs) on DPSC properties, a miRNA array was performed between dental periodontal ligament cells (DPLCs) and DPSCs. The results revealed that miR-224-5p (miR-224) was highly expressed in the DPSCs compared with that in the DPLCs. The transfection of DPSCs with an miR-224 inhibitor impaired cell viability. In addition, miR-224 inhibition significantly promoted cell apoptosis in DPSCscompared with the NC group. In silico analysis and a dual-luciferase reporter assay demonstrated that miR-224 targets the 3'-untranslated region of the Rac family small GTPase 1 (Rac1) gene. miR-224 downregulation resulted in the increased expression of Rac1 in DPSCs compared with DPLCs. Furthermore, miR-224 inhibition caused augmented mitogen-activated protein kinase 8 , caspase-3, caspase-9 and Fas ligand expression in DPSC, which may be recovered by Racl silencing with transfection with short hairpin RNA-Rac1. Furthermore, Annexin V-fluorescein isothiocyanate/propidium iodide flow cytometry indicated that the silencing of Rac1 restored the pro-apoptotic DPSC cell number with miR-224 transfection. Therefore, the results of the present study suggested miR-224 in DPSC serves an important function in protecting cells against apoptosis by downregulating Racl expression, and also identified miR-224 as a novel miRNA in regulating the features of DPSC.
\end{abstract}

\section{Introduction}

Stem cells are undifferentiated cells defined by their capacity for self-renewal, in addition to their ability to differentiate into

Correspondence to: Professor Jing Guo, Shandong Provincial Key Laboratory of Oral Tissue Regeneration, Department of Orthodontics, School of Stomatology, Shandong University, 107 Wenhua Xi Road, Jinan, Shandong 250012, P.R. China

E-mail: guojing@sdu.edu.cn; guojing_sdu@163.com

Key words: dental pulp stem cell, miR-224, Rac1, apoptosis other cell types $(1,2)$. Dental pulp stem cells (DPSCs) are a type of mesenchymal stem cell (MSC) in the cell-rich zone and core of the pulp tissue of teeth (3). DPSC possess strong self-renewal capabilities and the potential for multi-directional differentiation, rendering them with a high therapeutic potential for repairing damaged and/or defective tissues $(4,5)$. It has been documented that DPSCs are able to differentiate into multiple different cell lines, including osteoblasts, odontoblasts, chondrocytes, adipocytes, hair follicle cells, corneal endothelium cells, neuronal cells, melanocytes and endothelial cells (6-9).

MicroRNAs (miRs/miRNAs) are a group of non-coding RNA molecules, with an approximate length of 22-25 nucleotides, that are able to post-transcriptionally regulate the expression of their target genes, resulting in the degradation of the target mRNA (10). An increasing number of reports have indicated that miRNAs serve an essential function in numerous molecular and biological processes, including apoptosis, cell proliferation, migration and necrocytosis $(11,12)$. Frequently downregulated miR-224-5p (miR-224) expression in human tumor types has been demonstrated, and may possess fundamental functions in various cellular processes, particularly in modulating proliferation and differentiation (13-16). For instance, Li et al (17) suggested that miR-224, along with miR-21, may facilitate the osteogenic differentiation of periodontal ligament cells by targeting periodontal ligament associated protein-1 (17). miR-224 was also able to promote the osteogenic differentiation of human bone marrow MSCs, by targeting the enhancer of zeste 2 polycomb repressive complex 2 subunit/Wnt/ $\beta$-Catenin cascade (18). However, further studies are required to examine the underlying mechanism of miR-224 in regulating the function of human DPSCs.

Rac family small GTPase 1 (Rac1), accompanied by Ras homolog family member A and cell division cycle 42, belongs to the GTPase family, which controls the actin cytoskeleton accumulation and organization in mammalian cells and serves a critical signaling function in modulating various cellular processes $(19,20)$, including apoptosis, reactive oxygen species production, membrane ruffling, lamellipodia formation, the activity of transcriptional factors, cell cycle control and the integrity of cell-cell adhesions (21-26). Embade et al (27) reported the first evidence indicating that Racl induced apoptosis by a complex mechanism, involved the generation 
of ceramides and induced the de novo synthesis of Fas ligand (FasL). Another study also suggested that Racl mediated apoptosis via mitogen-activated protein kinase 8 (JNK) and served a key function in pro-apoptotic pathways in intestinal epithelial cells (28). The aim of the present study was to probe the function of miRs in the character of DPSCs, which may provide a new mechanism for regulating DPSC viability.

\section{Materials and methods}

Cell lines. DPSCs and dental periodontal ligament cells (DPLCs) were isolated from third molars or premolars extracted from at least 4 adults under the approved guidelines and protocol (ethically approved by the Ethics Committee of Shandong University, Shandong, China) with written informed consent obtained from all patients. The isolation and cultivation of human DPSCs and DPLCs were performed according to a previously reported method $(9,8)$. Briefly, tooth surfaces were cleaned and cut around the cementum-enamel junction by using sterilized dental fissure burs to reveal the pulp chamber. The pulp tissue was gently separated from the crown and root and then digested in a solution of $3 \mathrm{mg} / \mathrm{ml}$ collagenase type I (Worthington Biochemical Corporation, Lakewood, NJ, USA) and $4 \mathrm{mg} / \mathrm{ml}$ dispase (Boehringer Mannheim; Roche Applied Science, Mannheim, Germany) for $1 \mathrm{~h}$ at $37^{\circ} \mathrm{C}$. Single-cell suspensions were obtained by passing the cells through a $70-\mu \mathrm{m}$ strainer (Falcon ${ }^{\circledR}$; Corning, Inc.). Single-cell suspensions $\left(0.01-1 \times 10^{5} /\right.$ well $)$ of dental pulp and dental ligament cells were seeded into six-well plates (Costar; Thermo Fisher Scientific, Inc.) cultured in $\alpha$-Modified Essential Medium ( $\alpha$-MEM; Thermo Fisher Scientific, Inc., Waltham, MA, USA) supplemented with $15 \%$ fetal bovine serum (FBS; Thermo Fisher Scientific, Inc.), 2 mM L-glutamine (Thermo Fisher Scientific, Inc.), 100 units/ml penicillin (Sigma-Aldrich; Merck KGaA, Darmstadt, Germany) and $100 \mathrm{mg} / \mathrm{ml}$ streptomycin (Sigma-Aldrich; Merck KGaA) at $37^{\circ} \mathrm{C}$ under $5 \% \mathrm{CO}_{2}$ in air. All methods were approved by the Research Medical Ethics Committee of Shandong University (Shandong, China) and were performed in accordance with the approved guidelines.

For the induction of odentogenic differentiation of DPSCs, cells were grown in an osteoblast differentiating medium, consisting of $\alpha$-MEM supplemented with $10 \%$ FBS, $50 \mu \mathrm{g} / \mathrm{ml}$ ascorbic acid and $10^{-8} \mathrm{M}$ dexamethasone (Sigma-Aldrich; Merck KGaA).

miRNA array. Total RNA was extracted using the phenolchloroform method using TRIzol (Invitrogen; Thermo Fisher Scientific, Inc.). The quality of the RNA was assessed using capillary electrophoresis (Agilent Technologies, Inc., Santa Clara, CA, USA). Libraries for small RNA sequencing were prepared using the NEBNext Multiplex Small RNA Library Prep Set for Illumina (New England BioLabs, Inc., Ipswich, MA, USA) according to the manufacturer's protocol. The libraries were quantified using the Agilent Bioanalyzer 2100 system with DNA high-sensitivity chips. The raw sequence files were subjected to quality control analysis with the Fast QC quality control tool. To avoid low-quality data, adaptors were removed by Cutadapt (version 1.2.1; PyPI) and lower-quality sequences were trimmed. The clean reads were screened at a length of 21-22 nucleotides as miRNA and were located to the reference sequence using Bowtie software (version 2; CGE Risk Management Solutions B.V., Leidschendam, The Netherlands). The functions of novel miRNAs were analyzed using miRDeep2 software (version 2.0.0.8; Max Delbrück Center). Differential expression sequencing was used to calculate differential expression levels and to evaluate the statistical significance of detected alterations between the control and case samples.

Western blot analysis (WB). DPSCs and DPLCs were lysed in lysis buffer $(30 \mathrm{mM}$ Tris- $\mathrm{HCl}, 150 \mathrm{mM} \mathrm{NaCl}, 1 \% \mathrm{NP}-40$ and $0.1 \% \mathrm{SDS}$; pH 7.4) supplemented with a protease inhibitor cocktail (Roche Applied Science). The BCA Protein Quantitation kit (GenScript) was used to determine protein concentration. Proteins $(20 \mu \mathrm{g})$ were separated using 10\% SDS-PAGE and blotted electrophoretically onto polyvinylidene difluoride (Immobilon) membranes of $0.45 \mu \mathrm{m}$ pore size. Membranes were blocked with $5 \%$ bovine serum albumin (BSA) in phosphate-buffered saline containing $0.1 \%$ Tween-20 (PBST) for $1 \mathrm{~h}$ at room temperature, followed by incubation with the following primary antibodies overnight at $4^{\circ} \mathrm{C}$ : Anti-Rac1 antibody (1:4,000; cat no. ab33186; Abcam, Cambridge, UK), anti-JNK1 antibody (1:200; cat no. ab201624; Abcam), anti-caspase-3 antibody (1:1,000; cat no. ab4051; Abcam), anti-caspase-9 antibody $(1: 1,000$; cat no. ab25758; Abcam), anti-FasL antibody (1:2,000; cat no. ab15285; Abcam), anti-RUNX family transcription factor 2 (Runx2) antibody (1:1,500; cat no. ab23980; Abcam), anti-alkaline phosphatase (ALP) antibody (1:1,000; cat no. ab67228; Abcam), anti-actin antibody (1:5,000; cat no. ab1801; Abcam) and anti-GAPDH antibody (1:5,000; cat no. ab9485; Abcam). Horseradish peroxidase-conjugated goat anti-rabbit immunoglobulin G secondary antibodies (1:5,000; cat. no. AP132; Abcam) were incubated with the membranes for $1 \mathrm{~h}$ at room temperature in PBST containing 5\% BSA, followed by chemiluminescent detection. A C-DiGit Blot Scanner and Super Signal West Femto Maximum Sensitivity Substrate kit (Thermo Fisher Scientific, Inc.) were used to detect bound antibodies (PhotoShop CS6; Adobe Systems, Inc.).

RNA extraction and quantitative polymerase chain reaction (qPCR). Total RNA was extracted from DPSCs and DPLCs using TRIzol reagent (Invitrogen; Thermo Fisher Scientific, Inc.) according to the manufacturer's protocol (Invitrogen; Thermo Fisher Scientific, Inc.). mRNA levels were determined using the Light-Cycler 480 Real Time PCR system (Roche Applied Science), with GAPDH used as the internal control. Quantitative PCR was performed in $20 \mu \mathrm{l}$ reaction volumes containing SYBR-Green PCR Master Mix (Invitrogen; Thermo Fisher Scientific, Inc.) for $10 \mathrm{~min}$ at $95^{\circ} \mathrm{C} ; 95^{\circ} \mathrm{C}$ for $15 \mathrm{sec}, 60^{\circ} \mathrm{C}$ for $30 \mathrm{sec}$ and $72^{\circ} \mathrm{C}$ for $30 \mathrm{sec}$, for a total of 40 cycles. Transcript levels were determined relative to the endogenous reference gene (GAPDH), and normalized to the untreated sample (using the $2^{-\triangle \Delta C q}$ method). The expression of mRNA was first normalized to GAPDH, and the expression levels of miR-224 were first normalized to the endogenous control U6 small nuclear RNA. Then, the gene expression levels of genes in the treated group were compared with the level of gene expression in an untreated sample (29). 
Sequences of the primers used for qPCR detection were as follows: miR-224 forward, 5'-AGCCCCATCATCTACAGU G-3' and reverse, TCACAGTCACTAGGGCACC-3'; Rac1 forward, 5'-AAAATGTCCGTGCAAAGTGGT-3' and reverse, 5'-CTCGATCGTGTCTTTATCATCCC-3'; insulin like growth factor binding protein 5 (IGFBP-5) forward, 5'-TTGCCTCAA CGAAAAGAGC-3' and reverse, 5'-AGAATCCTTTGCGGT CACA-3'; JunB proto-oncogene, AP-1 transcription factor subunit (JunB) forward, 5'-CCAGTCCTTCCACCTCGACGT TTACAA-3' and reverse, 5'-GACTAAGTGCGTGTTTCT TTTCCACAG-3; nuclear receptor related-1 protein (NURR1) forward, 5'-GGCATGGTGAAGGAAGTTGT-3' and reverse, 5'-CAGGGAAGTGAGGAGATTGG-3'; GAPDH forward, 5'-GGAAGGTGAAGGTCGGAGTCA-3' and reverse, 5'-GTC ATTGATGGCAACAATATCCACT-3'; U6 forward, 5'-CTC GCTTCGGCAGCACA-3' and reverse, 5'-AACGCTTCACGA ATTTGCGT-3'.

miR-224 mimic/inhibitor preparation. Mimic/inhibitor of miR-224 and a negative control (NC) were acquired from Guangzhou RiboBio Co., Ltd. (Guangzhou, China). miR-224 mimic/inhibitor and NC mimic/inhibitor were supplemented with $0.9 \% \mathrm{NaCl}$ to a terminal level of $10 \mathrm{mg} / \mathrm{ml}$ for further use. Transfection of mimic/inhibitor was performed for $36 \mathrm{~h}$ at $37^{\circ} \mathrm{C}$.

RNA interference. Short hairpin RNAs (shRNAs) inhibiting Rac1 were generated via vector $\mathrm{pLKO} .1$ puro and oligonucleotides targeting the following Rac1 sequence: 5'-GATCCG GAAGGAGATTGGTGCTGTAAAACTCGAGAAAATGTC GTGGTTAGAGGAATTTTTG-3'. DPSCs and DPLCs cells were transiently transfected with pLKO.1-shRNA-Rac1 or pLKO.1 empty vector with Lipofectamine $2000^{\circledR}$ (Thermo Fisher Scientific, Inc.). After 48 h, the cells were washed with phosphate-buffered saline and harvested in lysis buffer [50 mM Tris-HCl (pH 7.4), 150 mM NaCl, 1 mM EDTA, $0.5 \mathrm{mM}$ dithiothreitol, $0.5 \%$ NP-40].

MTT assay. An MTT assay was conducted to evaluate cell proliferation. Briefly, DPSCs and DPLCs were treated with $20 \mu \mathrm{l}$ MTT $(0.5 \mathrm{mg} / \mathrm{ml})$, and the supernatant was discarded. Dimethyl sulfoxide $(150 \mu \mathrm{l})$ was then added to each well, with rotation for $10 \mathrm{~min}$, to dissolve the formazan dye. An Infinite M200 microplate reader (Tecan Group, Ltd., Männedorf, Switzerland) was then used to measure the absorbance at $490 \mathrm{~nm}$.

Colony formation assay. A colony formation assay was performed by seeding 1,000 of each DPSCs or DPLCs in $10-\mathrm{cm}^{2}$ flasks and culturing for 4 days. Cells were then washed with PBS and stained with $0.1 \%$ toluidine blue contained in $1 \%$ PFA overnight at $4^{\circ} \mathrm{C}$. On the following day, cells were washed to remove the excess dye. Stained clusters containing $>50$ cells were counted as positive colonies using an inverted light microscope (Leica MICROSYSTEMS; magnification, x40).

Terminal deoxynucleotidyl-transferase-mediated dUTP nick end labeling (TUNEL) staining. TUNEL staining was used to assess the degree of apoptosis in DPSCs and DPLCs using a TUNEL fluorescence kit (Roche Applied Science) according to the manufacturer's protocol. Briefly, cells were treated with $100 \mu \mathrm{l}$ TdT reaction buffer (Component A) and $50 \mu \mathrm{l}$ TdT reaction cocktail. Cells were then stained with $100 \mu$ l Andy Fluor ${ }^{\mathrm{TM}} 647$ (1:100 in staining buffer) for $30 \mathrm{~min}$. Subsequent to TUNEL staining, the cells were stained with DAPI solution for $15 \mathrm{~min}$ at $4^{\circ} \mathrm{C}(1: 5,000$; Beyotime Institute of Biotechnology, Haimen, China) to stain the nuclear DNA in antifade mouting medium. Fluorescence staining was viewed using a Laser Scanning Confocal Microscope (SP8; Leica Microsystems, Inc., Buffalo Grove, IL, USA). The apoptotic rate was calculated as TUNEL-positive cells in 10 fields.

Annexin V-fluorescein isothiocyanate(FITC)/propidium iodide (PI) flow cytometry $(F C)$. Cell death was evaluated using an Annexin V-FITC/PI apoptosis detection kit (cat. no. ab14085; Abcam), and the experiment was performed according to the manufacturer's protocol. Briefly, following transfection, cells were resuspended in $20 \mu \mathrm{l}$ binding buffer and then incubated for 20 min with $5 \mu \mathrm{l}$ PI and $10 \mu \mathrm{l}$ Annexin V-FITC in a dark room at $25^{\circ} \mathrm{C}$. Cell death was evaluated using BD FACSuite ${ }^{\mathrm{TM}}$ software (BD Biosciences).

Dual-luciferase reporter assay (DLRA). The 3'-untranslated region (UTR) of the Rac1 gene underwent amplification prior to fusion with the GV126 luciferase gene of pGL3 (Promega Corporation). The binding site of the Rac1 gene and miR-224 were mutated via site-directed mutagenesis, which served as a control. Thymidine kinase promoter (Takara Bio, Inc., Otsu, Japan; pRL-TK vectors) and plasmids containing Renilla luciferase were applied to adjust for transfection efficiency using Lipofectamine $2000^{\circledR}$ (Thermo Fisher Scientific, Inc.). 293 T cells (ATCC ${ }^{\circledR}$; CRL-3216 ${ }^{\mathrm{TM}}$ ) were co-transfected with miR-224-mimic and NC-mimic with luciferase reporter vectors, and the luciferase assay was conducted. Approximately $48 \mathrm{~h}$ after transfection, a dual-luciferase reporter gene assay kit (Promega Corporation) was used for detection before calculating the ratio of the firefly luciferase activity to Renilla luciferase activity.

TargetScan prediction. The prediction algorithm TargetScan was used to nominate targets of miR-224. Using the TargetScan Database predictions (http://www.targetscan.org) are ranked based on the predicted efficacy of targeting as calculated using cumulative weighted context++ scores of the sites (15). As an option, predictions are also ranked by their probability of conserved targeting (16).

Statistical analysis. All data are presented as the mean \pm standard deviation of three separate experiments. Data were analyzed using a Student's t-test for comparison between two groups or one way ANOVA with Tukey's post hoc test for comparison among multiple groups as appropriate. $\mathrm{P}<0.05$ was considered to indicate a statistically significant difference. Statistical analyses were performed using SPSS Statistics 17.0 (Version X; SPSS, Inc., Chicago, IL, USA).

\section{Results}

miR-224 is upregulated in DPSCs compared with DPLCs. As the high expression of Runx 2 protein is a marker of 
A

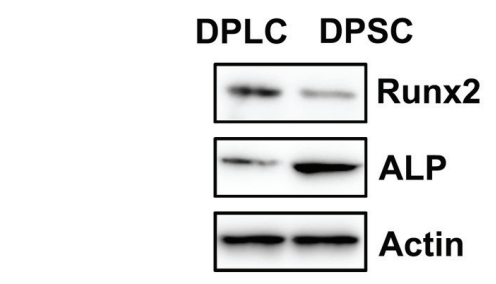

B

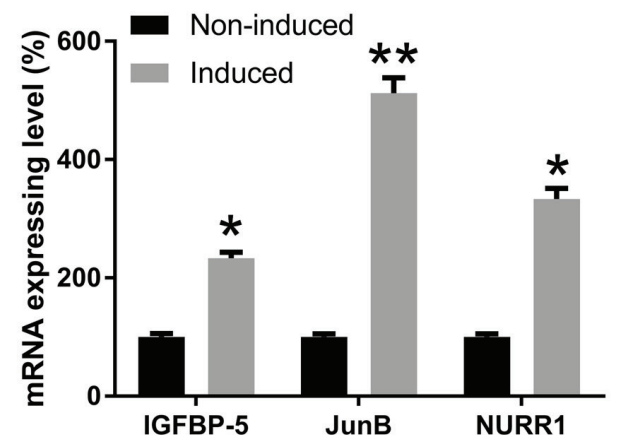

C

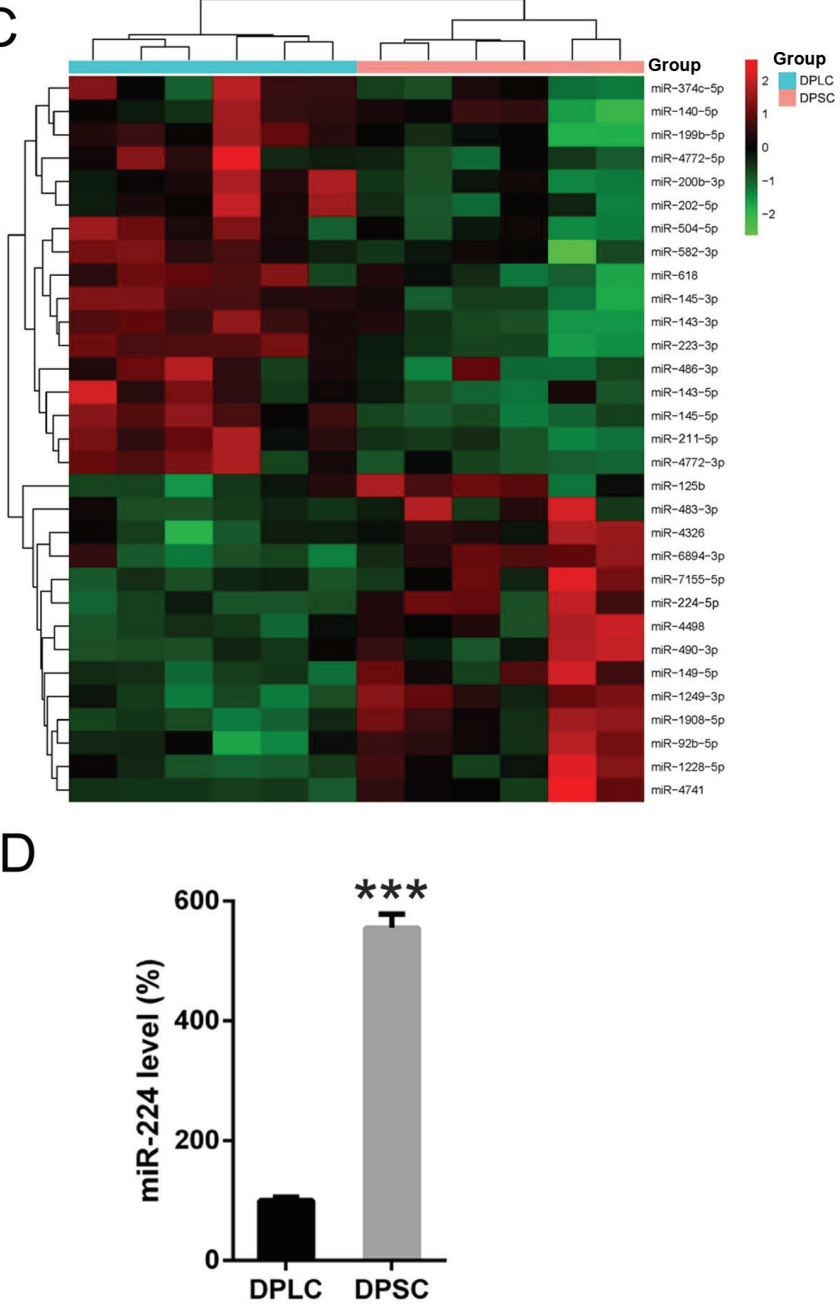

Figure 1. Expression of Runx2, ALP, IGFBP-5, JunB, NURR1 and miR-224 in DPLC and DPSC. (A) Western blot analysis was performed to assess the protein expression of Runx2 and ALP in DPLC and DPSC to confirm the successful isolation of the two cell lines. (B) DPSCs were grown in an osteoblast differentiating medium. A q-PCR was performed to detect the mRNA levels of IGFBP-5, JunB and NURR1. (C) A miRNA microarray of deregulated miRNAs in the DPLC and DPSC groups. (D) qPCR detection confirmed the expression of miR-224 in DPLC and DPSC. Mean \pm standard deviation of the results of three independent experiments was used to describe the data. $\mathrm{n}=3$. ${ }^{*} \mathrm{P}<0.05,{ }^{* * *} \mathrm{P}<0.01$ and ${ }^{* * * *} \mathrm{P}<0.001$, vs. the non-induced or DPLC group. miR/miRNA, microRNA; miR-224, miR-224-5p; DPSC, Dental pulp stem cells; DPLC, dental periodontal ligament cells; qPCR, quantitative polymerase chain reaction; Runx2, RUNX family transcription factor 2; ALP, alkaline phosphatase; IGFBP-5, insulin like growth factor binding protein 5; JunB, JunB proto-oncogene, AP-1 transcription factor subunit; NURR1, nuclear receptor related-1 protein.

DPLC (30), and DPSC typically displays a high expression of ALP protein subsequent to culturing (8), the present study examined the expression of these two proteins for further confirmation. As the results revealed, in the DPLC group, Runx2 protein expression was clearly higher compared with those in the DPSC group, while the DPSC group exhibited a higher ALP expression compared with the DPLC group (Fig. 1A). To examine the odentogenic differentiation property of DPSCs, DPSCs were grown in an osteoblast differentiating medium, consisting of $\alpha$-MEM supplemented with $10 \% \mathrm{FBS}, 50 \mu \mathrm{g} / \mathrm{ml}$ ascorbic acid and $10^{-8} \mathrm{M}$ dexamethasone. The present study then examined the mRNA levels of IGFBP-5, JunB and NURR1, which are three reported markers for differentiation. The mRNA levels of these three genes were significantly increased at day 10 with induction, compared with the non-induced group ( $\mathrm{P}<0.05$; Fig. 1B).

The miRNA microarray analysis revealed that miR-224 was substantially upregulated in DPSC, compared with DPLC (Fig. 1C). To confirm this data, qPCR analysis was performed

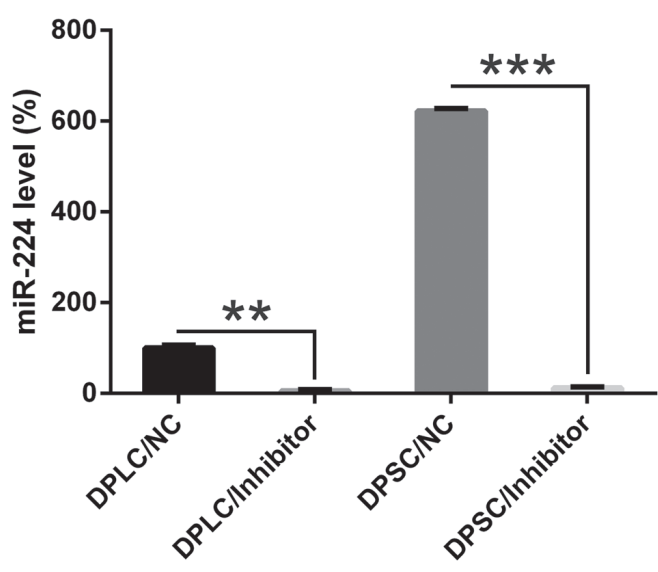

Figure 2. miR-224 inhibitor decreased the expression levels of miR-224 in the DPLC and DPSC groups compared with the NC. Mean \pm standard deviation of the results of three independent experiments were used to describe the data. $\mathrm{n}=3$. ${ }^{* *} \mathrm{P}<0.01$ and ${ }^{* * * *} \mathrm{P}<0.001$ with comparisons shown by lines. miRs, microRNAs; miR-224, miR-224-5p; DPSC, Dental pulp stem cells; DPLC, dental periodontal ligament cells; NC, negative control. 
A

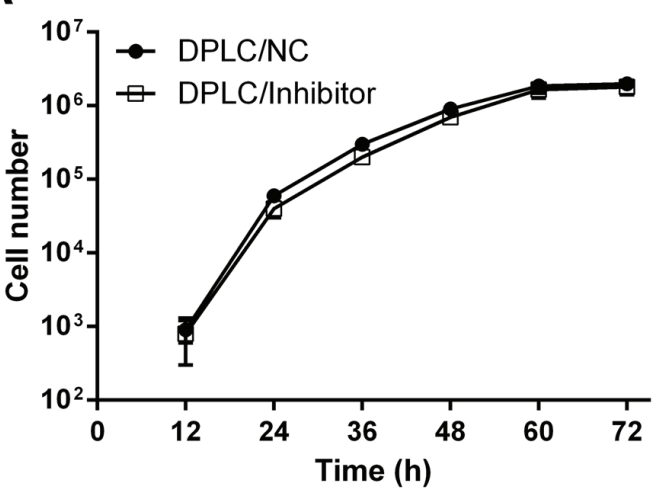

C
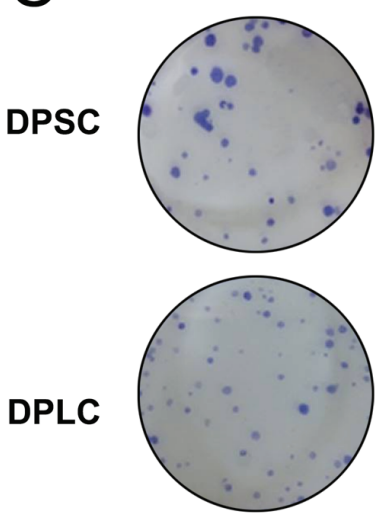

DPLC

Inhibitor

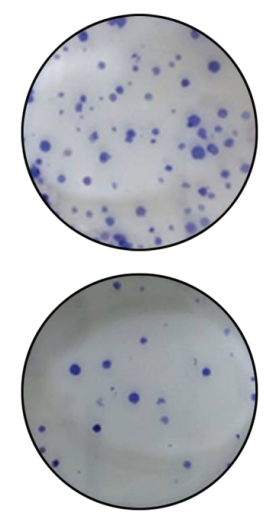

B

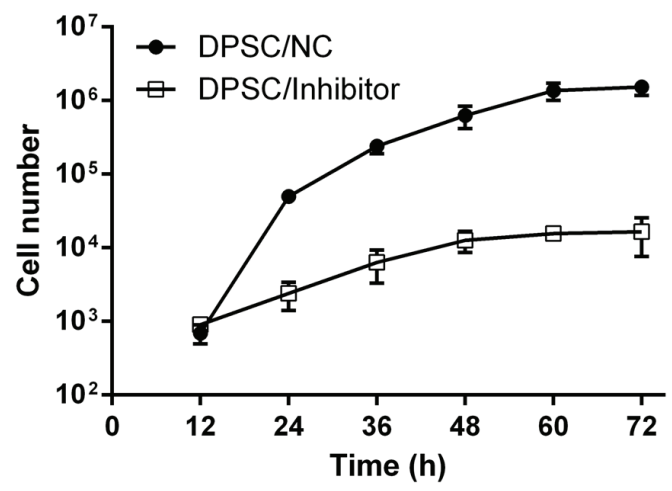

DPLC

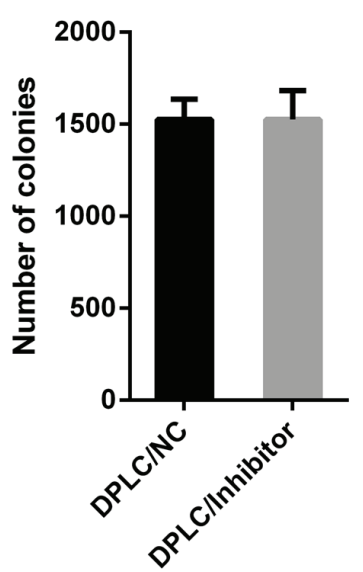

DPSC

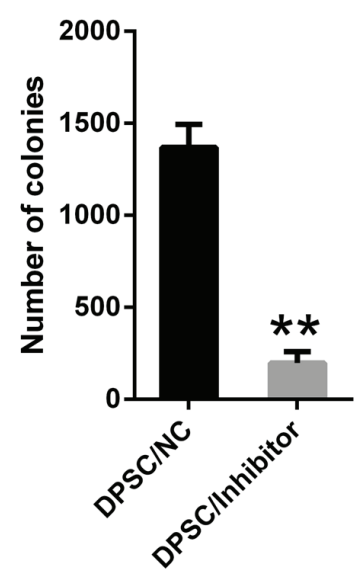

Figure 3. miR-224 inhibition affected DPSC proliferation. MTT assays were performed to examine the proliferation of (A) DPLC and (B) DPSC cells at 12-72 $\mathrm{h}$ subsequent to transfection. (C) A soft agar colony formation assay was performed to detect the colonies of the DPLCs and DPSCs transfected with the miR-224 inhibitor and $\mathrm{NC}$ inhibitor. Mean \pm standard deviation of the results of three independent experiments were used to describe the data. $\mathrm{n}=3$. ${ }^{* *} \mathrm{P}<0.01 \mathrm{vs}$. the $\mathrm{NC}$ group. miRs, microRNAs; miR-224, miR-224-5p; DPSC, dental pulp stem cells; DPLC, dental periodontal ligament cells; NC, negative control.

to determine miR-224 expression in DPLC and DPSC. It indicated that the expression levels of miR-224 were significantly upregulated in DPSC compared with DPLC $(\mathrm{P}<0.001$; Fig. 1D), suggesting that miR-224 may serve a functional role in DPSC properties.

miR-224 is essential for maintaining DPSC viability compared with DPLC viability. To elucidate the effect of miR-224 on the properties of DPLC and DPSC, the cells were transfected with miR-224 inhibitor to repress miR expression. The qPCR data indicated that using a miR-224 inhibitor resulted in significantly decreased miR-224 expression levels in DPLC and DPSC compared with the negative control ( $\mathrm{P}<0.01$; Fig. 2$)$. An MTT assay was subsequently performed to detect the proliferative rate of DPLC and DPSC during $72 \mathrm{~h}$ post-transfection. For DPLC, the data indicated that miR-224 depletion did not exert any effect (Fig. 3A), whereas miR-224 inhibition significantly suppressed the cell proliferation of DPSC during $72 \mathrm{~h}$ post transfection ( $\mathrm{P}=0.0236$; Fig. 3B). A colony formation assay was also performed to confirm the effect of miR-224 on cell viability, and the number of colonies of DPSC transfected with miR-224 inhibitor were reduced compared with DPLC transfected with miR-224 inhibitor, and significantly reduced compared with DPSC transfected with $\mathrm{NC}$ inhibitor $(\mathrm{P}<0.01$; Fig. 3C). These data suggested that miR-224 served a particularly critical function in sustaining DPSC viability compared with DPLC viability.

miR-224 inhibition induces the apoptosis of DPSC. It was hypothesized that miR-224 silencing may induce the apoptosis of DPSC, therefore causing attenuated DPSC viability. Thus, TUNEL staining and Annexin V-FITC/PI FC was conducted in two cell lines following the transfection of miR-224 inhibitor and NC inhibitor. DPSCs transfected with miR-224 inhibitor displayed significantly elevated numbers of cells with positive TUNEL staining at $36 \mathrm{~h}$ post transfection, compared with the DPSC and DPLC transfected with NC inhibitor $(\mathrm{P}<0.01$; Fig. 4A). Furthermore, Annexin V-FITC/PI FC demonstrated that silencing miR-224 expression significantly increased the percentage of apoptotic DPSC compared with DPLC $(\mathrm{P}<0.01$; Fig. 4B). These results suggested that miR-224 depletion resulted in an increased apoptotic percentage of DPSC.

miR-224 targets Racl. It has been reported that the Rac1 sensor serves an important function in modulating apoptotic cell signaling. Taking into consideration the key function of Rac1 
A
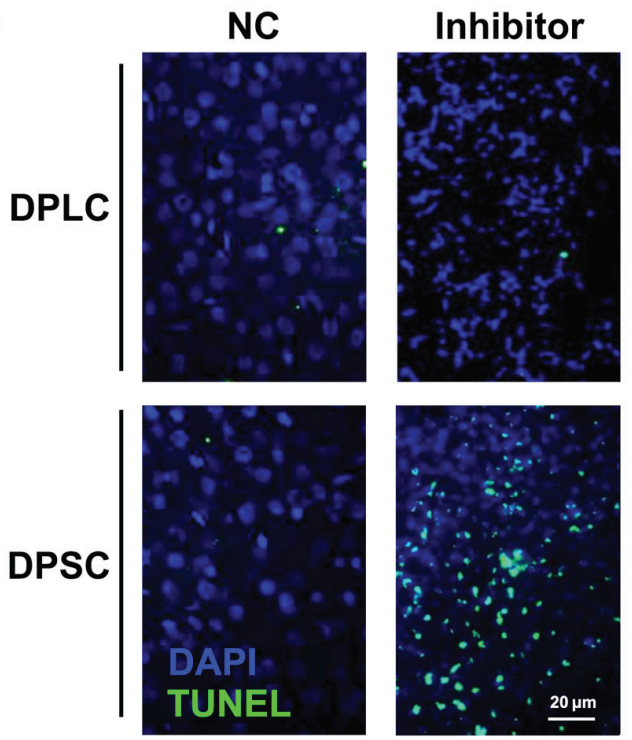

B
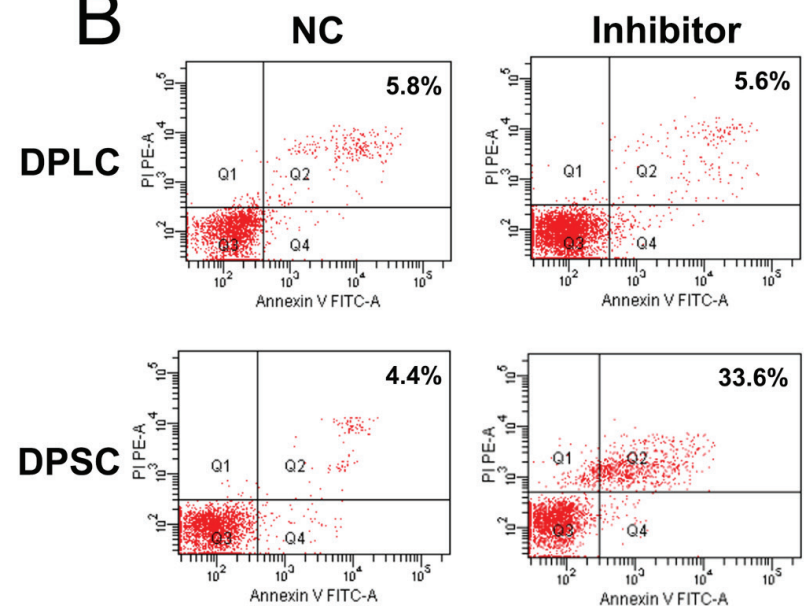

Annexin VFITC-A
DPLC

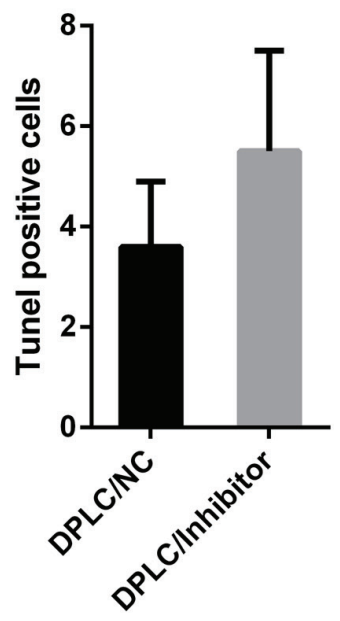

DPLC

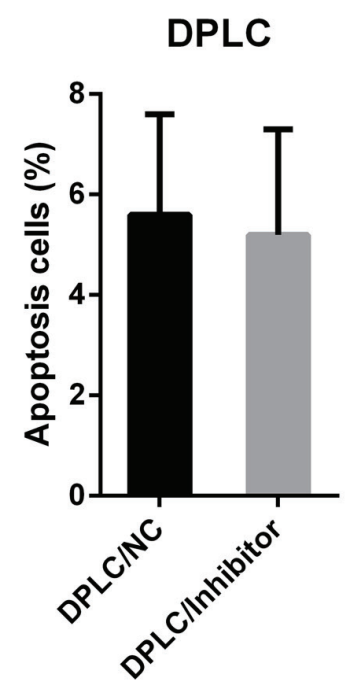

DPSC

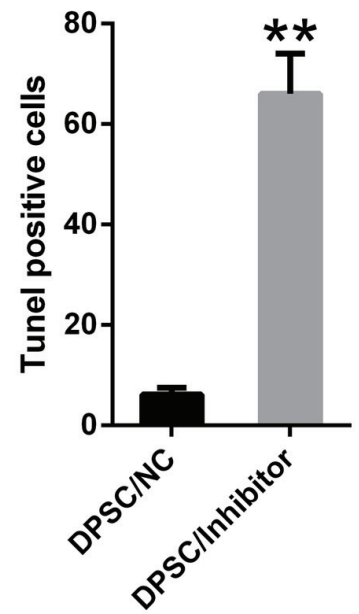

DPSC

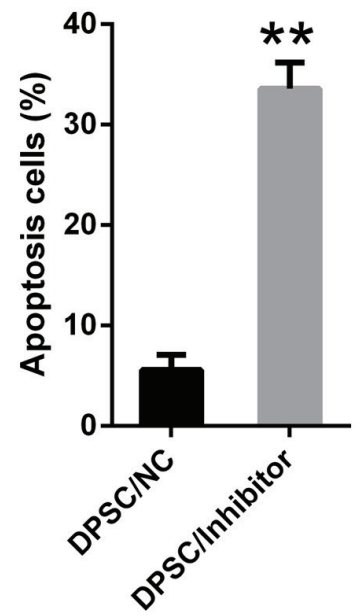

Figure 4. miR-224 inhibition enhanced the apoptosis of DPSC cells. (A) TUNEL staining was utilized in each group of DPLC and DPSC with miR-224 silencing or NC (magnification, x400). The apoptotic number of positive stained cells is displayed in the right panel. (B) Number of apoptotic cells, with early apoptotic cells presented in the right quadrant of each plot. Analysis of the apoptotic rate of the cells in all groups is displayed in the right panel. Mean \pm standard deviation of the results of three independent experiments were used to describe the data. $n=3$. ${ }^{* *} \mathrm{P}<0.01$ vs. the group. miRs, microRNAs; miR-224, miR-224-5p; DPSC, Dental pulp stem cells; DPLC, dental periodontal ligament cells; TUNEL, terminal deoxynucleotidyl-transferase-mediated dUTP nick end labeling; NC, negative control; FITC, fluorescein isothiocyanate; PI, propidium idodide.

protein in apoptosis, its expression was measured using WB and qPCR. The present study indicated the downregulation of Rac1 in DPSC compared with DPLC (Fig. 5A). In addition, Rac1 mRNA expression levels were significantly reduced in DPSC compared with DPLC $(\mathrm{P}<0.001$; Fig. 5B). Furthermore, bioinformatics analysis indicated that miR-224 may target the 3'-UTR of the Rac1 gene (Fig. 5C). Transfection with a miR-224 mimic significantly inhibited luciferase function compared with the negative control $(\mathrm{P}<0.01)$, which was fused with the Rac1 3'-UTR by $70 \%$ compared with the other control groups (Fig. 5D). Furthermore, the protein and mRNA expression levels of Rac1 were upregulated with miR-224 inhibitor transfection in DPSC cells, whereas Rac1 expression levels were not affected in transfected DPLC (Fig. 5E and F). These results suggest that miR-224 may target the 3'-UTR of Rac1.
Silencing of Racl suppresses the pro-apoptotic effect of miR-224 transfection in DPSC. To determine the effect of the miR-224 inhibitor and shRNA-Racl on the expression of miR-224 and Rac1 in DPSC, the present study detected the levels of miR-224 and Rac1 mRNA using qPCR analysis. The results revealed that miR-224 and Racl mRNA expression levels were significantly inhibited by transfections with miR-224 inhibitor and shRNA-Rac1, as compared with their respective negative control groups $(\mathrm{P}<0.01$; Fig. $6 \mathrm{~A}$ and $\mathrm{B})$. To determine whether Rac1 silencing may suppress the effect of miR-224 on apoptosis in DPSC, Racl was silenced in DPSCs transfected with miR-224 inhibitor or NC inhibitor. WB analysis was utilized in order to verify the changes in Racl expression levels (Fig. 6C). It was further indicated that the miR-224 inhibitor upregulated the expression of apoptotic 
A

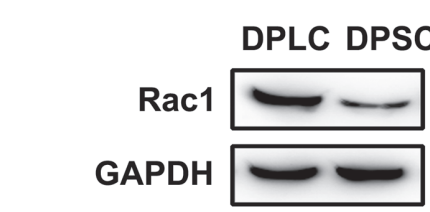

3'

miR-224

'uAcAguacuguga

I I I I I I I I

Rac1 WT ATGTCATGTTACAA

Rac1 MU GCTAGACTTTACAA

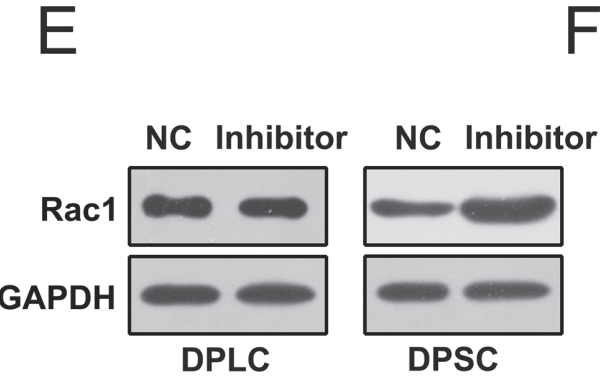

F
B

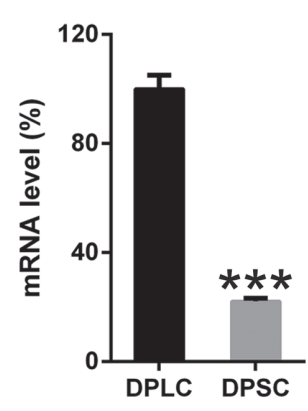

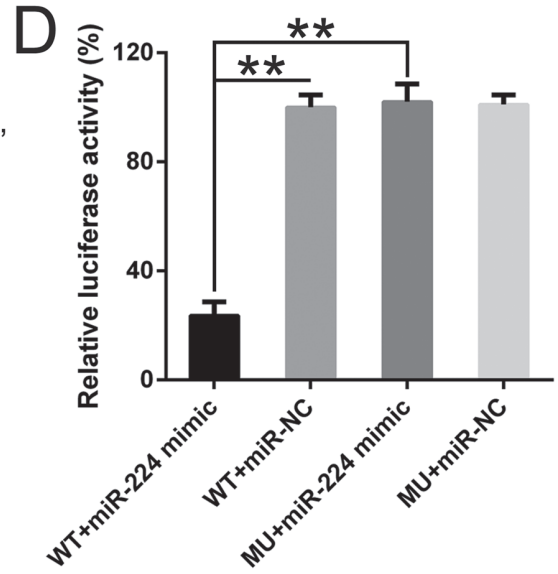

DPLC

DPSC
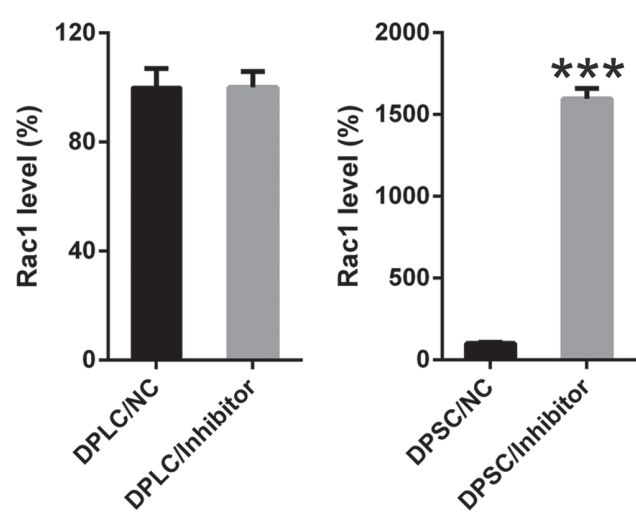

Figure 5. miR-224 targets the 3'-UTR of the Rac1 gene in DPSCs. (A) Western blot analysis and (B) quantitative polymerase chain reaction analysis determined the protein and mRNA expression levels of Rac1 in DPLCs and DPSCs. ${ }^{* * *} \mathrm{P}<0.001$ vs. the DPLC group (C) Bioinformatics analysis of miR-224 and the 3'-UTR of the Rac1 gene. (D) A dual-luciferase reporter assay was performed following co-transfection with a luciferase reporter containing either a WT or MU 3'-UTR from Rac1, and a miR-224-mimic into 293T cells. The effect of miR-224-mimic transfection on the luciferase activities of the WT and MU Rac1 reporter constructs was determined. ${ }^{* *} \mathrm{P}<0.01$ with comparisons shown by lines. Rac1 levels were determined at the (E) protein and (F) mRNA levels in DPLC and DPSC following transfection with an miR-224 inhibitor or an NC inhibitor. ${ }^{* * *} \mathrm{P}<0.001$ vs. the DPSC/NC group. Mean \pm standard deviation of the results of three independent experiments were used to describe the data. $n=3$. Rac1, Rac family small GTPase 1; WT, wild-type; MU, mutant; miRs, microRNAs; miR-224, miR-224-5p; DPSC, Dental pulp stem cells; DPLC, dental periodontal ligament cells; UTR, untranslated region; NC, negative control.

factors, including caspase-3, caspase-9 and particularly FasL, whereas the reduced expression of Rac1 decreased the pro-apoptotic effect of miR-224 inhibition in DPSC, as indicated by the reduced protein expression of caspase-3, caspase- 9 and FasL. It was reported that Racl promoted cell apoptosis through the JNK pathway; therefore, JNK1 expression was also detected in each group (31). WB data indicated that miR-224 inhibition or Rac1 upregulation resulted in increased JNK1 expression, while Rac1 downregulation decreased JNK1 expression. Annexin V-FITC/PI FC was performed to evaluate the apoptotic rate in DPSC with different treatments. Inhibition of miR-224 in DPSC resulted in a significant elevation in apoptotic cell proportion compared with the negative control $(\mathrm{P}<0.01)$, whereas Rac1 silencing significantly reduced the apoptosis of DPSC to a normal level compared with the miR-224 inhibitor treated group $(\mathrm{P}<0.01$; Fig. 6D).

\section{Discussion}

The aim of the present study was to examine the effect of miR-224 in human DPSCs, particularly its effect on cell proliferation and apoptosis. The expression of miR-224 in DPSCs was higher compared with that in DPLCs. In addition, miR-224 silencing in DPSCs, in contrast with DPLCs, caused impaired cell viability and promoted cell apoptosis. In silico prediction and DLRA data suggested that miR-224 targeted the 3'-UTR of 


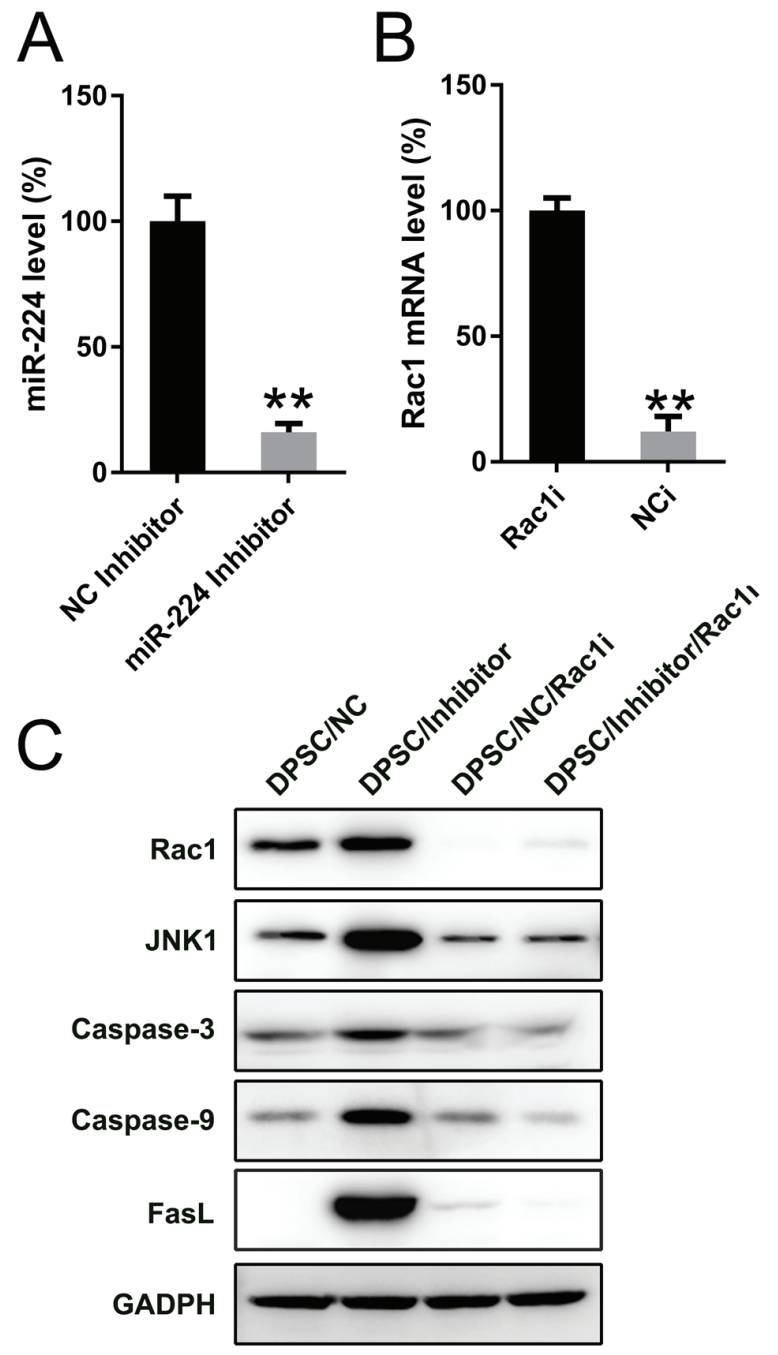

D
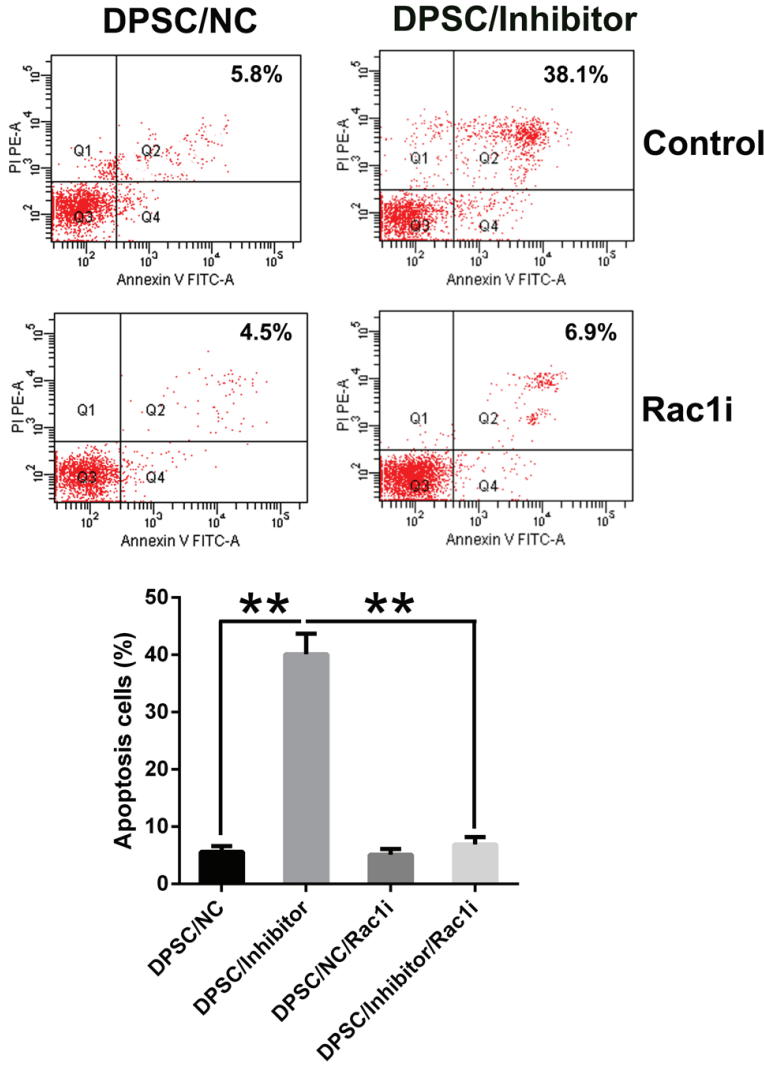

Figure 6. Rac1 silencing restored miR-224 inhibition impaired cell viability. DPSCs were transfected with either miR-224 inhibitor or shRNA-Rac1, or their controls. mRNA expression levels of (A) miR-224 and (B) Rac1 in each group were detected. ${ }^{* *} \mathrm{P}<0.01$ vs. the NC inhibitor group or the Rac1 inhibitor group, respectively. DPSCs were co-transfected with miR-224 inhibitor and shRNA-Rac1, or their controls. (C) Protein expression of Rac1, JNK1, caspase-3, caspase-9 and FasL in each group was detected. (D) Number of apoptotic cells. Early apoptotic cells are presented in the right quadrant of each plot. Analysis of the apoptotic rate of DPSCs in all groups is displayed in the lower panel. ${ }^{* *} \mathrm{P}<0.01$ with comparisons shown by lines. Mean \pm standard deviation of the results of three independent experiments were used to describe the data. $n=3$. Rac1, Rac family small GTPase 1; WT, wild-type; MU, mutant; miRs, microRNAs; miR-224, miR-224-5p; DPSC, Dental pulp stem cells; JNK1, mitogen-activated protein kinase 8; FasL, Fas ligand; shRNA, short hairpin RNA; FITC, fluorescein isothiocyanate; PI, propidium iodide.

the Racl gene, and the transfection of miR-224 mimic in DPSCs attenuated Racl expression at the protein and mRNA expression levels. The data also indicated that Rac1 silencing restored the expression of pro-apoptotic factors, including caspase-3 and FasL, and increased the apoptotic cell number, which were induced by miR-224 inhibition, thus serving as an essential target for miR-224 in maintaining cell viability.

The properties of human stem cells are regulated by intracellular or extracellular mechanical and molecular signals, and are a promising target for stem cell-based therapies for various types of human disease (32). However, the regulatory mechanisms of miRs on DPSC viability remain unclear. The results of the present study suggested that the high expression of miR-224 in DPSC promoted cell viability, as indicated by the increased number of apoptotic cells in the group transfected with an miR-224 inhibitor. However, a number of previous studies have reported contradictory results, suggesting that $\mathrm{miR}-224$ overexpression induces apoptosis in various cell lines. For instance, a retrospective report indicated that in neuroendocrine neoplasms, miR-224 agomir may promote apoptosis and suppress the proliferation and invasion of BON-1 cells (33). In SW480/adrenomedullin (ADM) cells, the transfection of an miR-224 inhibitor substantially increased glycogen synthase kinase-3 $\beta$ expression and decreased $\beta$-catenin and Survivin expression, resulting in enhanced cell apoptosis and suppressed ADM resistance (34). Nevertheless, additional studies have reported contradictory evidence and suggest that miR-224 inhibits the apoptosis of cells. Systemic lupus erythematosus (SLE) is a systemic autoimmune disease with abnormal T cell immune responses $(35,36)$. Chang et al $(37)$ reported that miR-145 and miR-224 were expressed aberrantly in SLE T cells that modulated the protein expression of their target genes, signal transducer and activator of transcription 1 (STAT1) and apoptosis inhibitor 5 (API5), respectively. These miRNA aberrations accelerated $\mathrm{T}$ cell activation-induced cell death by 
suppressing API5 expression and were associated with lupus nephritis by enhancing STAT1 expression (37). Another study demonstrated that amiodarone enhanced reactive oxygen species production and increased cell apoptosis, while it reduced DNA damage. Meanwhile, amiodarone suppressed miR-224 and increased its target cyclooxygenase-2 expression (37). For cervical cancer, Cong et al (38) indicated that the Notch1 gene is able to increase cervical cancer cell proliferation by regulating the miR-224/leucine rich repeats and immunoglobulin like domains 2 signal pathway. These controversial experimental evidence and conclusions may be attributed to the different cell lines applied in the studies, therefore indicating that miR-224 possesses a tissue- or cell-specific function in mediating apoptosis.

Previous evidence has indicated that Racl is able to regulate injury, damage, apoptosis and necrosis in various cell lines $(28,39,40)$. Jin et al $(28)$ revealed that Rac1 is activated during tumor necrosis factor- $\alpha$ (TNF- $\alpha)$-induced apoptosis in intestinal epithelial cells, and that the inhibition of Rac1, prior to the administration of apoptotic stimuli, substantially prevents apoptosis via the TNF- $\alpha$-induced activation of JNK1/2. Another study suggested that Rac 1 and Rac 2 stimulation mediated apoptosis through the apoptotic extrinsic pathway (Fas/FasL) (41). In the present study, inhibition of miR-224 increased the apoptotic level of DPSC, in addition to the expression levels of JNK1 and FasL, while Rac1 silencing restored the apoptosis and expression of these two proteins to normal levels. This suggests that the JNK pathway and Fas/FasL pathway participated in the apoptosis triggered by miR-224 inhibition.

In conclusion, these data indicate that miR-224 participates in the regulation of the cell viability of DPSC by directly repressing Rac1 levels and also indirectly, by inhibiting the expression of JNK and FasL, which may function as pro-apoptotic factors. The present study, therefore, suggests that miR-224 is a key modulator in maintaining the DPSC phenotype.

\section{Acknowledgements}

Not applicable.

\section{Funding}

The present study was supported by the Natural Science Foundation of Shandong Province (grant no. ZR2014HZ001).

\section{Availability of data and materials}

All data generated or analyzed during this study are included in this published article.

\section{Authors' contributions}

JG conceived and designed the study. WQ analyzed, interpreted data and drafted the manuscript. WQ, DL, QS, HuW and $\mathrm{HaW}$ performed the experiments. All authors participated in the writing and revision of the manuscript. DL and JG supervised the project. All authors read and approved the final manuscript.

\section{Ethics approval and consent to participate}

This study was approved by the Ethics Committee of Shandong University (Shandong, China) with written informed consent obtained from all patients.

\section{Patient consent for publication}

Not applicable.

\section{Competing interests}

The authors declare that they have no competing interests.

\section{References}

1. Sonoyama W, Liu Y, Fang D, Yamaza T, Seo BM, Zhang C, Liu H, Gronthos S, Wang CY, Wang S and Shi S: Mesenchymal stem cell-mediated functional tooth regeneration in swine. PLoS One 1: e79, 2006.

2. Gronthos S, Brahim J, Li W, Fisher LW, Cherman N, Boyde A DenBesten P, Robey PG and Shi S: Stem cell properties of human dental pulp stem cells. J Dent Res 81: 531-535, 2002.

3. Estrela C, Alencar AH, Kitten GT, Vencio EF and Gava E: Mesenchymal stem cells in the dental tissues: Perspectives for tissue regeneration. Braz Dent J 22: 91-98, 2011.

4. Iohara K, Nakashima M, Ito M, Ishikawa M, Nakasima A and Akamine A: Dentin regeneration by dental pulp stem cell therapy with recombinant human bone morphogenetic protein 2 . J Dent Res 83: 590-595, 2004.

5. Kerkis I, Kerkis A, Dozortsev D, Stukart-Parsons GC, Gomes Massironi SM, Pereira LV, Caplan AI and Cerruti HF: Isolation and characterization of a population of immature dental pulp stem cells expressing OCT-4 and other embryonic stem cell markers. Cells Tissues Organs 184: 105-116, 2006.

6. Gronthos S, Mankani M, Brahim J, Robey PG and Shi S: Postnatal human dental pulp stem cells (DPSCs) in vitro and in vivo. Proc Natl Acad Sci USA 97: 13625-13630, 2000.

7. Kawashima N: Characterisation of dental pulp stem cells: A new horizon for tissue regeneration? Arch Oral Biol 57: 1439-1458, 2012.

8. Mori G, Brunetti G, Oranger A, Carbone C, Ballini A Lo Muzio L, Colucci S, Mori C, Grassi FR and Grano M: Dental pulp stem cells: Osteogenic differentiation and gene expression. Ann N Y Acad Sci 1237: 47-52, 2011.

9. Mori G, Centonze M, Brunetti G, Ballini A, Oranger A, Mori C, Lo Muzio L, Tetè S, Ciccolella F, Colucci S, et al: Osteogenic properties of human dental pulp stem cells. J Biol Regul Homeost Agents 24: 167-175, 2010.

10. Bartel DP: MicroRNAs: Genomics, biogenesis, mechanism, and function. Cell 116: 281-297, 2004.

11. Wang XL, Zhang T, Wang J, Zhang DB, Zhao F, Lin XW, Wang Z, Shi P and Pang XN: MiR-378b promotes differentiation of keratinocytes through NKX3.1. PLoS One 10: e0136049, 2015.

12. Liu X, Gong J and Xu B: miR-143 down-regulates TLR2 expression in hepatoma cells and inhibits hepatoma cell proliferation and invasion. Int J Clin Exp Pathol 8: 12738-12747, 2015.

13. Lei Q, Shen F, Wu J, Zhang W, Wang J and Zhang L: MiR-101, downregulated in retinoblastoma, functions as a tumor suppressor in human retinoblastoma cells by targeting EZH2. Oncol Rep 32: 261-269, 2014

14. Strillacci A, Valerii MC, Sansone P, Caggiano C, Sgromo A, Vittori L, Fiorentino M, Poggioli G, Rizzello F, Campieri M and Spisni E: Loss of miR-101 expression promotes Wnt $/ \beta$-catenin signalling pathway activation and malignancy in colon cancer cells. J Pathol 229: 379-389, 2013.

15. Thu KL, Chari R, Lockwood WW, Lam S and Lam WL: miR-101 DNA copy loss is a prominent subtype specific event in lung cancer. J Thorac Oncol 6: 1594-1598, 2011.

16. Wang C, Lu S, Jiang J, Jia X, Dong $X$ and Bu P: Hsa-microRNA-101 suppresses migration and invasion by targeting Rac1 in thyroid cancer cells. Oncol Lett 8: 1815-1821, 2014. 
17. Li C, Li C, Yue J, Huang X, Chen M, Gao J and Wu B: miR-21 and miR-101 regulate PLAP-1 expression in periodontal ligament cells. Mol Med Rep 5: 1340-1346, 2012.

18. Wang H, Meng Y, Cui Q, Qin F, Yang H, Chen Y, Cheng Y, Shi J and Guo Y: MiR-101 Targets the EZH2/Wnt/ $\beta$-catenin the pathway to promote the osteogenic differentiation of human bone marrow-derived mesenchymal stem cells. Sci Rep 6: 36988, 2016.

19. Hall A: G proteins and small GTPases: Distant relatives keep in touch. Science 280: 2074-2075, 1998.

20. Hall A: Rho GTPases and the actin cytoskeleton. Science 279: 509-514, 1998.

21. Coso OA, Chiariello M, Yu JC, Teramoto H, Crespo P, Xu N, Miki T and Gutkind JS: The small GTP-binding proteins Racl and Cdc42 regulate the activity of the JNK/SAPK signaling pathway. Cell 81: 1137-1146, 1995.

22. Knaus UG, Heyworth PG, Evans T, Curnutte JT and Bokoch GM: Regulation of phagocyte oxygen radical production by the GTP-binding protein Rac 2. Science 254: 1512-1515, 1991.

23. Minden A, Lin A, Claret FX, Abo A and Karin M: Selective activation of the JNK signaling cascade and c-Jun transcriptional activity by the small GTPases Rac and Cdc42Hs. Cell 81 1147-1157, 1995.

24. Moore KA, Sethi R, Doanes AM, Johnson TM, Pracyk JB, Kirby M, Irani K, Goldschmidt-Clermont PJ and Finkel T: Rac1 is required for cell proliferation and $\mathrm{G} 2 / \mathrm{M}$ progression. Biochem J 326: 17-20, 1997.

25. Ridley AJ, Paterson HF, Johnston CL, Diekmann D and Hall A: The small GTP-binding protein rac regulates growth factor-induced membrane ruffling. Cell 70: 401-410, 1992.

26. Takaishi K, Sasaki T, Kotani H, Nishioka H and Takai Y: Regulation of cell-cell adhesion by rac and rho small $\mathrm{G}$ proteins in MDCK cells. J Cell Biol 139: 1047-1059, 1997.

27. Embade N, Valerón PF, Aznar S, López-Collazo E and Lacal JC: Apoptosis induced by Rac GTPase correlates with induction of FasL and ceramides production. Mol Biol Cell 11: 4347-4358, 2000.

28. Jin S, Ray RM and Johnson LR: Rac1 mediates intestinal epithelial cell apoptosis via JNK. Am J Physiol Gastrointest Liver Physiol 291: G1137-G1147, 2006.

29. Livak KJ and Schmittgen TD: Analysis of relative gene expression data using real-time quantitative PCR and the 2(-Delta Delta C(T)) method. Methods 25: 402-408, 2001.

30. An S, Huang X, Gao Y, Ling J, Huang Y and Xiao Y: FGF-2 induces the proliferation of human periodontal ligament cells and modulates their osteoblastic phenotype by affecting Runx2 expression in the presence and absence of osteogenic inducers. Int J Mol Med 36: 705-711, 2015.
31. Zhang Y, Dai Q, Zeng F and Liu H: MALAT1 promotes the proliferation and metastasis of osteosarcoma cells by activating the Rac1/JNK pathway via targeting miR-509. Oncol Res: Apr 27, 2018 (Epub ahead of print). doi: 10.3727/096504017X1 4957939026111.

32. Hassan MQ, Tye CE, Stein GS and Lian JB: Non-coding RNAs: Epigenetic regulators of bone development and homeostasis. Bone 81: 746-756, 2015.

33. Bai J, Na H, Hua X, Wei Y, Ye T, Zhang Y, Jian G, Zeng W, Yan L and Tang Q: A retrospective study of NENs and miR-224 promotes apoptosis of BON-1 cells by targeting PCSK9 inhibition. Oncotarget 8: 6929-6939, 2017.

34. Liang CQ, Fu YM, Liu ZY, Xing BR, Jin Y and Huang JL: The effect of miR-224 down-regulation on SW80 cell proliferation and apoptosis and weakening of ADM drug resistance. Eur Rev Med Pharmacol Sci 21: 5008-5016, 2017.

35. Suárez-Fueyo A, Bradley SJ and Tsokos GC: T cells in systemic lupus erythematosus. Curr Opin Immunol 43: 32-38, 2016.

36. Lu MC, Lai NS, Chen HC, Yu HC, Huang KY, Tung $\mathrm{CH}$, Huang HB and Yu CL: Decreased microRNA(miR)-145 and increased miR-224 expression in T cells from patients with systemic lupus erythematosus involved in lupus immunopathogenesis. Clin Exp Immunol 171: 91-99, 2013.

37. Chang YL, Liu ST, Wang YW, Lin WS and Huang SM: Amiodarone promotes cancer cell death through elevated truncated SRSF3 and downregulation of miR-224. Oncotarget 9: 13390-13406, 2018.

38. Cong L, Zhang $\mathrm{F}$ and Shang $\mathrm{H}$ : Notch1 targeted regulation of mir-224/LRIG2 signaling for the proliferation and apoptosis of cervical cancer cells. Oncol Lett 13: 2304-2308, 2017.

39. Shen E, Li Y, Li Y, Shan L, Zhu H, Feng Q, Arnold JM and Peng T: Racl is required for cardiomyocyte apoptosis during hyperglycemia. Diabetes 58: 2386-2395, 2009.

40. Yoshida T, Zhang Y, Rivera Rosado LA, Chen J, Khan T, Moon SY and Zhang B: Blockade of Racl activity induces G1 cell cycle arrest or apoptosis in breast cancer cells through downregulation of cyclin D1, survivin, and X-linked inhibitor of apoptosis protein. Mol Cancer Ther 9: 1657-1668, 2010.

41. Gulbins E, Coggeshall KM, Brenner B, Schlottmann K, Linderkamp $\mathrm{O}$ and Lang F: Fas-induced apoptosis is mediated by activation of a ras and rac protein-regulated signaling pathway. J Biol Chem 271: 26389-26394, 1996.

(i) $(9)$ This work is licensed under a Creative Commons Attribution-NonCommercial-NoDerivatives 4.0 International (CC BY-NC-ND 4.0) License. 